\title{
On Cyclical Phase Transformations in Driven Alloy Systems
}

\begin{abstract}
JONG K. LEE
Cyclical phase transformations occurring in driven materials syntheses such as ball milling are described in terms of a free energy minimization process of participant phases. The oscillatory flow behavior of metals with low stacking fault energies during hot working is taken as a prototype in which a ductile crystalline phase sustains undulation in its free energy, due to the alternate succession of work-hardening and work-softening mechanisms. A time-dependent, oscillatory free energy function is then obtained by solving a delay differential equation (DDE), which accounts for a time lag due to diffusion. To understand cyclical transitions on an atomistic scale, work is extended to molecular dynamics simulations. Under shear deformation, a two-dimensional nanocrystal shows cyclical transitions between an equilibrium rhombus and a nonequilibrium square phase. Three-dimensional simulations show crystalline-to-glass transitions at high strain rates, but very high shear strain rates are found to lead to a latticelike network structure in the plane perpendicular to the shear direction, with strings of atoms parallel to the shear direction.
\end{abstract}

DOI: $10.1007 / \mathrm{s} 11661-007-9379-\mathrm{z}$

(C) The Minerals, Metals \& Materials Society and ASM International 2007

\section{INTRODUCTION}

MATERIAL synthesis via external dynamic forcing is called a "driven process." Perhaps, the best known driven process is mechanical alloying - a high-energy ball-milling process - but other examples include severe plastic deformation and irradiation. An interesting feature observed in several driven processes is temporal oscillations in phase fractions. For example, during mechanical alloying, the microstructure of a binary alloy of elemental $\mathrm{Al}_{50} \mathrm{Zr}_{50}$ powders is shown to vary cyclically between a crystalline and an amorphous state, ${ }^{[1]}$ while Co powders are shown to display changes between a fcc and a hep structure. ${ }^{[2]}$ In order to shed some light on the mechanism(s) of such cyclical phase transformations, we present a few findings obtained with both thermodynamic and atomic modeling approaches.

Mechanical alloying is a nonequilibrium thermodynamic process used to synthesize a variety of both equilibrium and nonequilibrium materials; examples include nanocrystalline solids, intermetallics, and glassy alloys. From a thermodynamic viewpoint, a system under external dynamic forcing experiences continuous energy input, and neither the formation of nonequilibrium structures nor the recovery of equilibrium structures through the decomposition of nonequilibrium structures is unexpected. Temporal oscillations in phase structure (or in any other property) under driven conditions are phenomena that occur far from equilib-

JONG K. LEE, Professor, is with the Department of Materials Science and Engineering, Michigan Technological University, Houghton, MI 49931, USA. Contact e-mail: jk1103@mtu.edu

This article is based on a presentation given in the symposium entitled "Solid-State Nucleation and Critical Nuclei during First Order Diffusional Phase Transformations," which occurred October 15-19, 2006 during the MS\&T meeting in Cincinnati, Ohio under the auspices of the TMS/ASMI Phase Transformations Committee.

Article published online December 8, 2007 rium. Consequently, the term "equilibrium," used frequently to describe the microstructure, is incorrect, as the system would continue to evolve even if the external driving force were removed. Whether a driven system undergoes sustained oscillations or reaches a steadystate microstructure, the total free energy, including that of the loading system, tends toward a minimum.

A survey of some observed temporal oscillations in property under driven conditions ${ }^{3,4]}$ is provided in Table I. The first four alloy systems show cyclical phase transformations between a crystalline and a glass phase at high milling intensity. At low milling intensity, however, alloy Nos. 1 and 4 show a stable glass phase upon milling crystalline powders. Cyclical variations in hardness for elemental Zn (No. 7) and in stored enthalpy for $\mathrm{Cu}$ and austenitic steels (Nos. 8 through 10) suggest that a ductile crystalline phase could oscillate in its free energy under a given driven condition. This is the hypothesis on which this work is prescribed, and through which all the phenomena listed in Table I are elucidated. In Section II, a delay differential equation (DDE) is used, to explore a thermodynamic base for cyclical phase transformations; in Section III, molecular dynamics simulations are attempted, to understand the cyclical transition on an atomic scale. No distinction is made between amorphous and glassy states. Further, the word "cyclic" is used loosely, to indicate temporal oscillations with or without a definite period; therefore, it could mean either decaying or sustained oscillations.

\section{THE DDE APPROACH}

\section{A. Oscillatory Flow Behavior during Hot Working}

Hot working is an important thermomechanical process in which many metals and alloys are worked 
Table I. Systems with Cyclical Variations in Property under Driven Conditions

\begin{tabular}{|c|c|c|c|c|}
\hline Number & Alloy System & Dynamic Force & Property & Observations \\
\hline 1 & $\mathrm{Al}_{50} \mathrm{Zr}_{50}$ & mechanical alloying ${ }^{[1]}$ & structure & $\mathrm{C} \leftrightarrow$ A cyclic phase transformations* \\
\hline 2 & $\mathrm{Co}_{50} \mathrm{Ti}_{50}$ & mechanical alloying ${ }^{[5]}$ & structure & $\mathrm{C} \leftrightarrow \mathrm{A}$ cyclic phase transformation \\
\hline 3 & $\mathrm{Co}_{3} \mathrm{Ti}$ & mechanical alloying ${ }^{[6]}$ & structure & $\mathrm{C} \leftrightarrow \mathrm{A}$ cyclic phase transformation \\
\hline 4 & $\mathrm{Cu}_{33.3} \mathrm{Zr}_{66.7}$ & mechanical alloying ${ }^{[7]}$ & structure & $\mathrm{C} \leftrightarrow \mathrm{A}$ cyclic phase transformation \\
\hline 5 & $\mathrm{Cu}_{33.3} \mathrm{Zr}_{66.7}$ & electron irradiation ${ }^{[8]}$ & structure & $\mathrm{C} \leftrightarrow \mathrm{A} \leftrightarrow \mathrm{C}$ with increase in dose \\
\hline 6 & $\mathrm{Co}$ & mechanical alloying $^{[2]}$ & structure & hcp + fcc $\leftrightarrow$ hcp $\leftrightarrow$ fcc + hcp $\leftrightarrow$ fcc \\
\hline 7 & $\mathrm{Zn}$ & mechanical alloying (cryogenic) ${ }^{[9]}$ & hardness & three decaying cycles between 0.8 and $0.3 \mathrm{GPa}$ \\
\hline 8 & $\mathrm{Cu}$ & mechanical alloying $^{[10]}$ & enthalpy & two cycles between 0.4 and $1.2 \mathrm{~kJ} / \mathrm{mole}$ \\
\hline 9 & $\mathrm{Cu}$ & hot torsion ${ }^{[11]}$ & torque & cyclic variations between 0.38 and $0.52 \mathrm{~N} \cdot \mathrm{m}$ \\
\hline 10 & austenitic steel & hot torsion ${ }^{[12]}$ & stress & cyclic variations between 14 and $35 \mathrm{MPa}$ \\
\hline
\end{tabular}

into a final or an intermediate product at high temperatures. Metals such as copper or austenitic steels with low stacking fault energies feature both diffusional flow and dislocation motion during hot working. As such, the true stress-true strain relationship depends on the strain rate (hereupon, the use of the term "true" for both stress and strain is dropped). At low strain rates, the stress-strain curve displays an oscillatory behavior with multiple peaks. As the strain rate increases, the number of peaks on the stress-strain curve decreases and, at high strain rates, the stress rises to a single peak before reaching a steady-state value. It is understood that dynamic recovery is responsible for the stress-strain behavior with zero or a single peak, whereas dynamic recrystallization causes the oscillatory nature. In the past, most predictive models are based on either modified Johnson-Mehl-Avrami kinetic equations or probabilistic approaches. ${ }^{[13-20]}$ In this work, a DDE is used for modeling this type of stress-strain behavior: a delay time due to diffusion is taken into consideration, and it is expressed in terms of a critical strain for nucleation for recrystallization.

For alloys with low stacking fault energies, both cross slip and climb are difficult, due to large stacking faults, and this, in turn, reduces the rate of dynamic recovery through dislocation motion. ${ }^{[17]}$ Consequently, the dislocation densities can reach the critical level for the onset of recrystallization. At low strain rates, there is sufficient time for the recrystallizing grains to grow before they become saturated with high dislocation densities. The flow curve then becomes oscillatory, due to recurrent cycles of recrystallization. With an increase in the strain rate, however, the difference in stress between recrystallizing and old grains diminishes, resulting in a reduced driving force for grain growth and a rendering of smaller grains in the alloy. Therefore, there emerge fewer peaks with concurrent deformation, due to high strain rates. Eventually, there will be a single peak at a sufficiently high stain rate. In a temporal sense, microstructural evolution at low strain rates is inhomogeneous, but becomes progressively homogeneous as the stain rate increases.

Let us consider a ductile polycrystalline phase such as copper, capable of storing a form of mechanical energy.
According to Kocks, ${ }^{[21]}$ the rate of change in dislocation density, $\rho$, with respect to strain, $\varepsilon$, may be given by

$$
\frac{d \rho}{d \varepsilon}=k_{1} \sqrt{\rho}-k_{2} \rho
$$

where $k_{1}=$ the rate of dislocation storage and $k_{2}=$ the rate of dislocation recovery. Using the familiar relationship between stress and dislocation density, $\sigma=a \mu \sqrt{\rho}$, Eq. [1] can be converted to an equation for the rate of change in stress with respect to strain:

$$
\frac{d \sigma}{d \varepsilon}=0.5 a \mu k_{1}-0.5 k_{2} \sigma
$$

where $\mu$ is the shear modulus and $a$ is a material constant. If we take both $k_{1}$ and $k_{2}$ as constants, Eq. [2] yields, as expected, a solution in a simple exponential form:

$$
\sigma=a \mu k_{1} / k_{2}+\left(\sigma_{0}-a \mu k_{1} / k_{2}\right) \exp \left(-0.5 k_{2} \varepsilon\right)
$$

where $\sigma_{0}$ is the initial stress at $\varepsilon=0$. With Eq. [3], $\sigma$ increases monotonically from $\sigma_{0}$ to the steady-state value, $\sigma_{s s}=a \mu k_{1} / k_{2}$. We note that Eq. [2] is based on the assumption that the recovery occurs instantaneously and the magnitude of softening, i.e., recovery, depends only on the current stress, $\sigma$, at $\varepsilon$. Therefore, it is no surprise to see that Eq. [2] fails to exhibit multiple peaks in the stress-strain curve. One could contemplate a strain-dependent $k_{1}$ (or $k_{2}$ ) in Eq. [2] and search for a solution, but it will not be a fertile effort, since little is understood about either $k_{1}$ or $k_{2}$, although the ratio, $k_{1} / k_{2}$, may be estimated from $\sigma_{s s}$.

As noted before, diffusional flow is part of deformation process during hot working. If an incubation time is needed for the nucleation of new recrystallizing grains, for the climb of dislocations or for some other event, the current rate of recovery might depend on the deformation state at an earlier time, as well as on the current state of deformation. Under deformation at a constant strain rate, $\dot{\varepsilon}$, an incubation time, $\tau$, can be related to an incubation strain, $\varepsilon_{n}$, for example, through $\varepsilon_{n}=\dot{\varepsilon} \tau$. In order to investigate the influence of an incubation strain on the stress-strain relationship, we now introduce a DDE of the form 


$$
\frac{d \sigma}{d \varepsilon}=f\left\{\sigma(\varepsilon), \sigma\left(\varepsilon-\varepsilon_{1}\right), \sigma\left(\varepsilon-\varepsilon_{2}\right), \ldots\right\}
$$

where $\varepsilon_{1}, \varepsilon_{2}$, etc., are incubation strains characteristic of the alloy recovery process. Thus, the rate of recovery depends on the current stress $\sigma$ evaluated at $\varepsilon$, well as on earlier stresses evaluated at $\varepsilon-\varepsilon_{1}, \varepsilon-\varepsilon_{2}$, etc. For simplicity, let us consider a single delay strain, $\varepsilon_{n}$, and write

$$
\frac{d \sigma}{d \varepsilon}=\theta-\frac{\theta}{\omega} \sigma\left(\varepsilon-\varepsilon_{n}\right)
$$

Appropriately, in the limit of $\varepsilon_{n}=0$, Eq. [5] becomes identical to Eq. [2], with $\theta=0.5 a \mu k_{1}$ and $\omega=\sigma_{s s}=$ $a \mu k_{1} / k_{2}$. Equation [5] can be solved either numerically or analytically, by using the same initial condition. Similar to the ordinary differential case of Eq. [2], we take

$$
\sigma(\varepsilon)=\sigma_{0} \quad \text { for } \varepsilon \leq 0
$$

Substituting Eq. [6] into Eq. [5] gives

$$
\frac{d \sigma}{d \varepsilon}=\theta-\frac{\theta}{\omega} \sigma_{0} \quad \text { for } 0 \leq \varepsilon<\varepsilon_{n}
$$

which is integrated to yield

$$
\sigma(\varepsilon)=\sigma_{0}-\frac{\theta}{\omega}\left(\sigma_{0}-\omega\right) \varepsilon \quad \text { for } 0 \leq \varepsilon \leq \varepsilon_{n}
$$

For the next period, Eq. [5] becomes

$$
\frac{d \sigma}{d \varepsilon}=\theta-\frac{\theta}{\omega}\left[\sigma_{0}-\frac{\theta}{\omega}\left(\sigma_{0}-\omega\right)\left(\varepsilon-\varepsilon_{n}\right)\right] \text { for } \varepsilon_{n} \leq \varepsilon<2 \varepsilon_{n}
$$

Integrating Eq. [9] from $\varepsilon_{n}$ to $\varepsilon$ and using $\sigma\left(\varepsilon_{n}\right)$ at $\varepsilon=\varepsilon_{n}$ from Eq. [8]:

$$
\begin{gathered}
\sigma(\varepsilon)=\sigma_{0}-\frac{\theta}{\omega}\left(\sigma_{0}-\omega\right) \varepsilon+\left(\frac{1}{2}\right)\left(\sigma_{0}-\omega\right)\left\{\frac{\theta}{\omega}\left(\varepsilon-\varepsilon_{n}\right)\right\}^{2} \\
\text { for } \varepsilon_{n} \leq \varepsilon<2 \varepsilon_{n}
\end{gathered}
$$

Continuing the same iterative integrations for higher strain intervals yields

$$
\begin{gathered}
\sigma(\varepsilon)=\sigma_{0}+\left(\sigma_{0}-\omega\right) \sum_{m=1}^{p} \frac{(-\theta / \omega)^{m}\left\{\varepsilon+(1-m) \varepsilon_{n}\right\}^{m}}{m !} \\
\quad \text { for }(p-1) \varepsilon_{n} \leq \varepsilon<p \varepsilon_{n}
\end{gathered}
$$

In the limit of $\varepsilon_{n}=0$, the summation term in Eq. [11] is equal to $\exp (-\theta \varepsilon / \omega)-1$, and thus Eq. [11] becomes identical to Eq. [3], which should be no surprise. If the hardening rate term is dropped in Eq. [5], Eq. [11] is also identical to the result of Epstein, ${ }^{[22]}$ who investigated the utility of DDE in examining various chemical kinetics.

The flow behavior of Eq. [11] is presented in Figure 1, in which $\omega$ is varied from 3,2 , and 1.5 to 1 , for a case with $\theta=4, \varepsilon_{n}=0.3$, and $\sigma_{0}=0$. Two distinct features can be noticed. First, as $\omega$ decreases, the flow curve begins to oscillate with an increasing number of stress peaks. Second, regardless of oscillations, the stress approaches a steady-state value set equal to $\omega$. A stability analysis shows that when $\lambda \quad\left(=\theta \varepsilon_{n} / \omega\right) \leq$ $1 / e=0.37, \sigma$ increases monotonically from $\sigma_{o}$ to $\sigma_{s s}=$ $\omega$. When $1 / e<\lambda<\pi / 2=1.57$, however, the stress displays an oscillatory behavior with a decaying amplitude. At $\lambda=\pi / 2$, the oscillation becomes perpetual, with a period equal to $4 \varepsilon_{n}$. If $\lambda>\pi / 2$, it is pulsating with a growing amplitude, suggesting an "accelerated softening." For our purposes, $\sigma(\varepsilon)$ with $\lambda \leq \pi / 2$ seems physically relevant.

In their classic work, ${ }^{[13]}$ Luton and Sellars correctly proposed that multiple stress peaks should appear when the ratio of the characteristic strain for nucleation of recrystallization, $\varepsilon_{n}$, to the characteristic strain for completion of recrystallization, $\varepsilon_{x}$, is large. If $\omega / \theta$ is taken to be $\varepsilon_{x}$ on the basis that $\theta$ is a high-temperature modulus and $\omega$ is a steady-state stress, the ratio, $\varepsilon_{n} \varepsilon_{x}$, is then equivalent to $\lambda=\theta \varepsilon_{n} / \omega$. This ratio is the reason the stress starts to display an oscillatory behavior when $\lambda$ is greater than a critical value, $1 / e=0.37$. Low strain rates provide adequate time for the recrystallizing grains to grow before they become saturated with high dislocation densities. The final (average) grain size at the steady state increases with a decrease in the strain rate, resulting in a lower $\sigma_{s s}$. Thus, $\omega$ should decrease with a decrease in the strain rate, $\dot{\varepsilon}$. Taking temperature effects into consideration, let us utilize a creep powerlaw type and write $\omega=A \dot{\varepsilon}^{q} \exp (q Q / \mathrm{R} T)=\gamma \dot{\varepsilon}^{q}$, where $Q$ is the activation energy for self diffusion and $q \sim 0.2 .^{[23]}$ As an example, with $\gamma=4$ and $q=0.2, \omega$ is converted to yield $\dot{\varepsilon}$, which is shown in the last column of Figure 1.

We now apply Eq. [11] to the flow behavior of a plain 0.25 pct $\mathrm{C}$ steel in the austenitic state (fcc) at $1100{ }^{\circ} \mathrm{C}$ $\left(0.76 T_{m}\right)$. The solid curves shown in Figure 2 are the stress-strain curves obtained from torsion tests, ${ }^{[12,23]}$ and the dotted curves are the DDE results fitted to the experimental data. The input parameters used for the DDE results are given in Table II. ${ }^{[24]}$ For comparison, the $\gamma$ values are also evaluated from $\omega / \dot{\varepsilon}^{0.2}$ with the experimental strain rates, $\dot{\varepsilon}$. The average values of $\theta, \varepsilon_{n}$, and $\gamma$ are listed in the bottom row. Ideally, the DDE

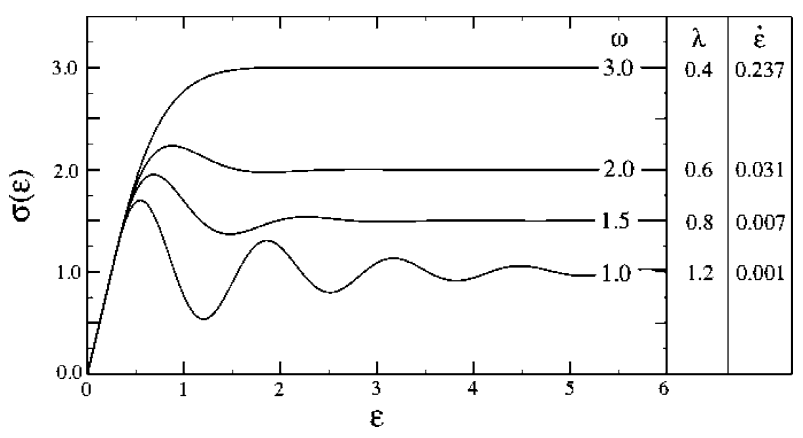

Fig. 1-Stress-strain relationship according to the solution of a DDE (Eq. [5]). The $\omega$ is varied from 3,2, and 1.5 to 1 , for a case with $\theta=4, \varepsilon_{n}=0.3$, and $\sigma_{o}=0$. The $\lambda$ values are equal to $\theta \varepsilon_{n} / \omega$. The strain rates, $\dot{\varepsilon}$, are obtained from a creep power-law relationship, $\omega=4 \dot{\varepsilon}^{0.2}$. 


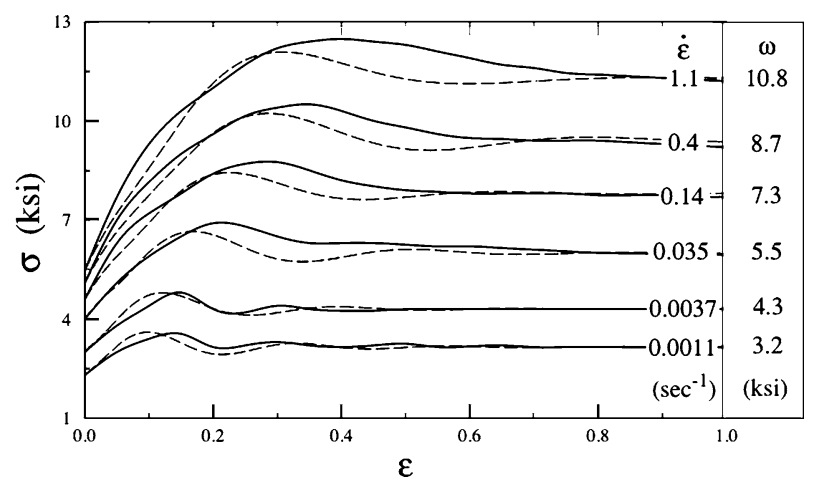

Fig. 2 - Comparison of flow curves. Solid curves: torsional flow behavior of a plain 0.25 pct $\mathrm{C}$ steel in the austenitic state, at $1100{ }^{\circ} \mathrm{C}$ from Rossard. ${ }^{[2,23]}$ Dotted curves: DDE-predicted curves with Eq. [11].

prediction should be made with a constant for each of $\theta$ and $\varepsilon_{n}$, and then should yield a constant for $\gamma$. This is not the case, and thus the comparison between the torsion tests and the DDE prediction is fair, at best. However, we note substantial variations involved in getting experimental data: among others, they can be initial sample conditions, conversion to true stress and true strain, strain-dependent grain size at the steady state, etc. For example, different $\sigma_{0}$ values suggest that the torsion samples did not have the identical initial condition, such as the same average grain size, at the beginning. Because of these variations, there is a lag in the first stress peak position between the DDE and the experiment. Another reason for the lag can be attributed to the softening rate coefficient, $\theta / \omega$, in Eq. [5]: the experimental curves indicate a faster coefficient, $\varphi / \omega$, with $\varphi>\theta$. The $\lambda$ and $\tau$ will be discussed later. It seems fair to conclude that the DDE model, expressed in Eq. [5], displays most of the basic physics associated with the flow behavior of metals with low stacking fault energies during hot working.

\section{B. Cyclical Phase Transformations between Crystalline and Glass Phase}

During hot working, metals experience a rolling power (energy/volume/time) that is proportional to the strain rate. A fraction of the rolling power is converted to stored mechanical energy, and much is dissipated as heat. The efficiency depends on the material and the rolling device, and defects such as dislocations or grain boundaries represent the stored mechanical energy.
In addition, there occurs recovery, such as dislocation annihilation or recrystallization of new grains. Thus, the thermodynamic state of the deforming metal should be expressed in terms of free energy, which requires the detailed nature of the crystalline defects and the entropic state of the metal, both configurational and vibrational. To a first-order approximation, however, the true stress, $\sigma$, should be a good representation for the free energy per unit volume, $G$. In developing Eq. [11], $\sigma=\mathrm{a} \mu \rho^{0.5}$ as used, but if other defects such as grain boundaries are taken into account, the approximation seems reasonable, provided that there arise no significant temperature changes that might cause transitions in the crystal structure.

Thus replacing $\sigma$ and $\varepsilon$ with $G$ and $\dot{\varepsilon} \tau$, respectively, Eq. [11] is transformed to yield a time-dependent free energy, $G(t)$ :

$$
\begin{gathered}
G(t)=G_{0}+\left(G_{0}-G_{s s}\right) \sum_{m=1}^{n} \frac{(-\xi)^{m}\{t+(1-m) \tau\}^{m}}{m !} \\
\qquad \text { for }(n-1) \tau \leq t<n \tau
\end{gathered}
$$

where $G_{s s}\left(=\sigma_{s s}=\omega\right)$ is the steady-state or saturated free energy, $\tau$ is the incubation time necessary for recovery due to diffusion, and $\xi(=\theta \dot{\varepsilon} / \omega)$ represents the rate of material recovery. The advantage of Eq. [12] compared to Eq. [11] is that it can be applied to other driven processes in which its input energy power can be controlled. For example, the free energy of copper during ball milling can be viewed with Eq. [12]. In ball milling, the milling intensity is roughly equal to the product of the angular momentum of the vial and the charge ratio, i.e., the ratio of the grinding media mass to the alloy powder mass. ${ }^{[3]}$ Therefore, under a constant milling intensity process, the time, $t$, indicates the milling time.

Let us apply Eq. [12] to cyclical phase transformations observed during ball milling. As Courtney and Lee ${ }^{[3]}$ pointed out, we may classify the crystalline-glass transition into three groups: (1) stable crystalline phase state, (2) cyclical transition between crystalline and glass phase, and (3) stable glass phase state upon transition of an initially crystalline phase. Let us now release the implicit assumption used for the derivation of Eq. [12], that regardless of the state of $G(t)$, the activation free energy required for the $\alpha$ to $\beta$ (or any other different phase) transition is prohibitively large. Furthermore, we imagine that the transition is instantaneous whenever the free energy of the $\alpha$ phase is greater than that of the $\beta$ phase (that is, some degree of supersaturation required

Table II. Input and Output Parameters for the DDE Curves in Figure 2

\begin{tabular}{llcccccc}
\hline$\omega(\mathrm{ksi})$ & $\sigma_{0}(\mathrm{ksi})$ & $\theta(\mathrm{ksi})$ & $\varepsilon_{n}$ & $\dot{\varepsilon}\left(\mathrm{s}^{-1}\right)$ & $\gamma=\omega / \dot{\varepsilon}^{0.2}$ & $\lambda\left(=\theta \varepsilon_{n} / \omega\right)$ & $\tau\left(=\varepsilon_{n} / \dot{\varepsilon}\right)$ \\
\hline 10.8 & 5.5 & 75.0 & 0.1 & 1.1 & 10.6 & 0.69 & 0.09 \\
8.7 & 5.1 & 75.5 & 0.1 & 0.4 & 10.4 & 0.87 \\
7.3 & 4.6 & 73.5 & 0.08 & 0.14 & 10.8 & 0.81 \\
5.5 & 4.0 & 73.0 & 0.07 & 0.035 & 10.8 & 0.93 \\
4.3 & 3.0 & 70.0 & 0.055 & 0.0037 & 13.2 & 0.90 \\
3.2 & 2.3 & 65.0 & 0.05 & 0.0011 & 12.5 & 1.02 & 14.9 \\
- & (average) & $(72.0)$ & $(0.076)$ & - & $(11.4)$ & 45.5 \\
\hline
\end{tabular}


for transition is neglected). The instantaneity suggests no kinetic barriers. Likewise, the same assumption applies for the reverse transition, from $\beta$ to $\alpha$.

Figure 3 illustrates case (b), with cyclical phase transitions between a ductile crystalline $\alpha$ and a glass $\beta$ phase. The two free energy curves are constructed with $\xi=1.3$ for $\alpha$ and $\xi=0.3$ for $\beta$, and an incubation period of $\tau=1$ is taken for both phases. The ground state free energy of the $\beta$ phase, $G_{0 \beta}$, is taken to be about $1.7 \mathrm{G}_{0 \alpha}$, a comparable level such that an extensive competition between two phases would occur during milling. If a phase is unable to work harden or to recover, its free energy would reach an asymptotic value without oscillations. Although a glass phase is known to deform through shear banding, its free energy "pulse" should be much weaker when compared to that of a ductile crystalline phase. For this reason, a small $\xi$ is assigned for $\beta$. Alternatively, with the aid of Figure 1, this case could be viewed with a small $\tau$ for $\beta$ and a large $\tau$ for $\alpha$ at a fixed $\xi$. Some binary alloy systems, such as $\mathrm{Al}_{50} \mathrm{Zr}_{50}$ or $\mathrm{Co}_{50} \mathrm{Ti}_{50}$ (when milled at high intensity), should belong to this case.

Obviously, if $G_{0 \beta}$ is far greater than $G_{0 \alpha}$, as marked with $\mathbf{a}$ in Figure 3, the two free energy curves never cross each other, and the system should remain in a crystalline $\alpha$ phase. Milling elemental metal powders such as $\mathrm{Cu}$ or $\mathrm{Zn}$ should belong to this class. Conversely, if $G_{0 \beta}$ is close to (but still greater than) $G_{0 \alpha}$, for example, to the level marked with $\mathbf{c}$ in Figure 3, we should expect to observe a stable $\beta$ phase, except for the onset of milling with the initial " $\alpha$ " powders. Some alloys, such as $\mathrm{Al}_{50} \mathrm{Zr}_{50}$ and $\mathrm{Cu}_{33.3} \mathrm{Zr}_{66.7}$ (Nos. 1 and 4 in Table I), show glass phases at low milling intensity but cyclical phase transformations between crystalline and glass phase at high milling intensity. Thus, Eq. [12] can describe why such interesting changes would occur with different milling intensities.

The milling of elemental Co powders (No. 6 in Table I) presents quite a different feature: cyclical transformations between two crystalline phases, hcp and fcc, or a mixture of both. Figure 4 could explain why such a case is tenable. The values of $\xi$ and $\tau$ are 1.3 and 1.0 , respectively, for $\alpha$, and 1.4 and 0.8 , respectively, for $\phi$. Therefore, both phases are oscillatory, but with

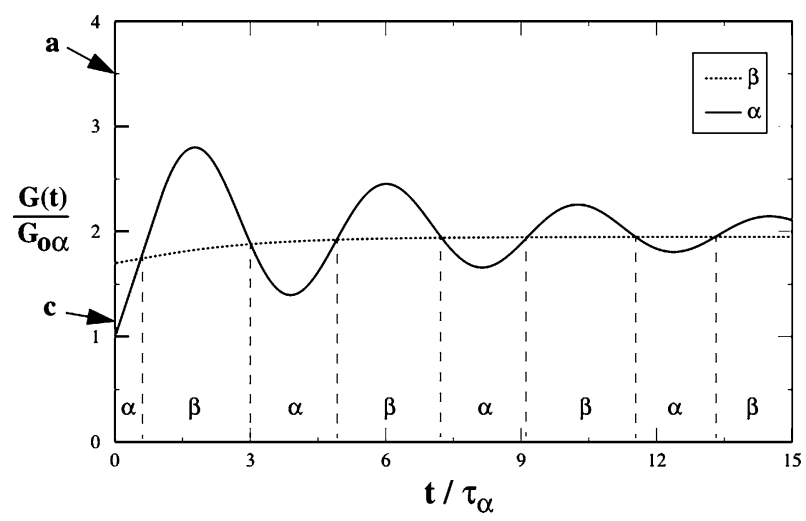

Fig. 3-Free energy states for cyclical transitions between a crystalline $\alpha$ and a glass $\beta$ phase.

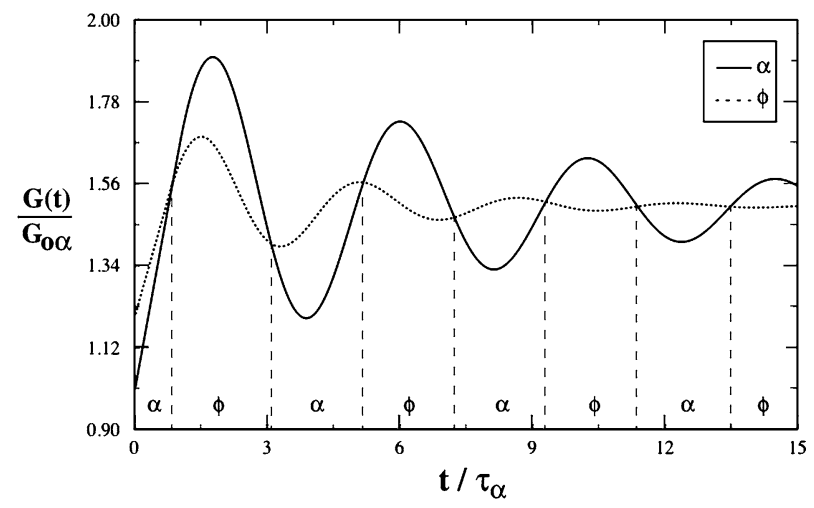

Fig. 4 -Free energy states for cyclical transitions between two crystalline phases, $\alpha$ and $\phi$.

different periods and amplitudes. If $\alpha$ and $\phi$ are taken to be hcp and fcc, respectively, Figure 4 demonstrates the possibility of cyclical transformations between two crystalline phases.

In describing the results of Figures 3 and 4, we have assumed no kinetic barriers for phase transitions. With more realistic kinetic barriers, oscillations are expect to decay faster and the system should early on reach a dynamic equilibrium at which the phase fractions attain their steady-state values. ${ }^{[3]}$ The DDE model of Eq. [12] is a linear approximation with one single delay. Clearly, one may attempt to include multiple delays or nonlinear terms, or a mix of both, and could expect more interesting results, although the mathematical complexity increases. Finally, it should be noted that, in a driven process, the ground-state free energy density, $G_{0}$, of a phase is not necessary equal to the value at bulk. For example, some amorphous nanoparticles are shown to have higher stability, because melting points often decrease with particle size. ${ }^{[25]}$

\section{NONEQUILIBRIUM MOLECULAR DYNAMICS STUDIES}

\section{A. Two-Dimensional Case}

A simulation work via molecular dynamics is performed on a two-dimensional, model nanocrystal that undergoes a martensitic-like transition between a hightemperature square phase (S) and a low-temperature rhombus phase (R). Figure 5 shows snapshot pictures for both S and R crystallite made of 57 Lennard-Jones (LJ) atoms. The crystallites are a modified version of Kastner's 41-atom nanocrystal, which was studied for shape-memory effects. ${ }^{[26,27]}$ The $12-6 \mathrm{LJ}$ potential of an atom interacting with $\mathrm{N}$ other atoms is given by

$$
\Phi_{i}=\sum_{j=1}^{N} 2 \varepsilon_{i j}\left\{\left(\frac{\sigma_{i j}}{r_{i j}}\right)^{12}-\left(\frac{\sigma_{i j}}{r_{i j}}\right)^{6}\right\}
$$

Both the potential parameters and the atomic masses are listed in Table III. All units are reduced with characteristic length $\sigma=10^{-10} \mathrm{~m}$, mass $M=5.8 \times 10^{-26} \mathrm{~kg}$, 


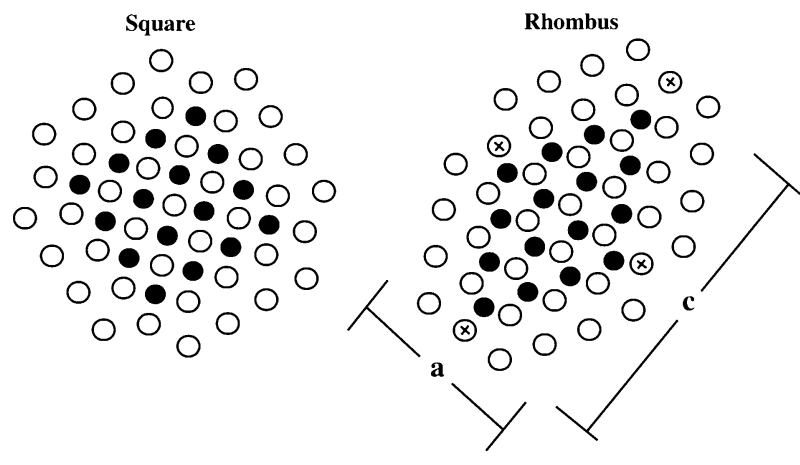

Fig. 5- Snapshot pictures of a square and a rhombus phase. The a and $\mathbf{c}$ indicate the minor and major axes between the $\oplus$ marked $\mathrm{A}$ atoms.

energy $\varepsilon=2.5 \times 10^{-19} \mathrm{~J}$, or time $\tau=4 \times 10^{-14} \mathrm{~s}=$ $0.04 \mathrm{ps}$ ( $\mathrm{ps}=$ a picosecond, $10^{-12}$ seconds). If we ignore the 16 white atoms (A) located as a perimeter layer in Figure 5, the inner 41 atoms form a binary ordered structure. As the black atoms (B) are smaller and much lighter than A atoms, the structure can be regarded as an interstitially ordered phase. Kastner ${ }^{[26]}$ found that, at low temperatures, the rhombus, i.e., $\mathrm{R}$ phase is thermodynamically stable, with six nearest-neighbor-like atoms.

At high temperatures, however, due to vibrational entropies, the $\mathrm{S}$ phase becomes stable, with four nearestneighbor-like atoms. At the absolute zero, the atomic volume of the $\mathrm{S}$ phase is greater than that of the $\mathrm{R}$ phase by 7 pct. In order to enhance the stability of both phases and to prevent phase decomposition into pure $\mathrm{A}$ and pure $\mathrm{B}$ during a thermomechanical processing, the $16 \mathrm{~A}$ atoms are fenced around Kastner's original 41-atom cluster. The free energy difference between the two structures is found by calculating the isothermal strain energy associated with the elastic deformation between the two structures. From the free energy information, the transition temperature, $T_{c}$, between the $\mathrm{R}$ and the $\mathrm{S}$ phases is found to be $\sim 791 \mathrm{~K} .{ }^{[28]}$

Figure 6 presents the structural changes of the nanocrystal as a function of "milling" time, under a periodic impact of shear deformation. The external loading is a pure shear of 4 pct, imparted at a time interval of $10 \tau$. The shear is performed along an arbitrary line passing through the center of mass of the crystal. A scaled velocity Verlet algorithm is employed, to follow the trajectories of the atoms. ${ }^{[27]}$ The structure is monitored with the change in the aspect ratio, $c / a$, i.e., the ratio of the major to the minor axis. The aspect ratio of the $\mathrm{R}$ phase fluctuates about 1.6, while the $S$ phase fluctuates about 1.1. During milling, the temperature is shown to maintain below $650 \mathrm{~K}$ through quenching. Here, "temperature" means an average value for 500 time-steps, with each step equal to $0.01 \tau$. Since the shear impact heats up the crystal, it is quenched at the rate of $560 \mathrm{~K} / \mathrm{ps}$, as long as its temperature is above $400 \mathrm{~K}$. With its temperature below $T_{c}=791 \mathrm{~K}$, the crystal maintains $\mathrm{R}$ phase during most of the milling period. However, the $\mathrm{S}$ phase is shown to be visible, though intermittent.
Figure 6(b) presents the details between milling times 15,800 and 16,000 , during which a cycle of R-to-S-to-R transitions is shown. In fact, the structures shown in Figure 5 are the snapshots at the time of 15,820 for $R$ and 15,902 for $\mathrm{S}$, respectively.

The oscillations with a time interval of about $10 \tau$ shown in Figure 6(b) are due to the periodic impact of shearing deformation. Although the average temperature is maintained below the transition temperature, $791 \mathrm{~K}$, the crystal is often heated up close to the transition temperature upon the shear impact. Depending on the impact orientation, shear deformation can cause displacive atomic motions, which in turn lead to structural changes. Let us examine how collective atomic motions lead to the structural changes. In Figure 7, the head of each "tadpole" indicates atomic positions at the milling time of 15,830 , whereas the tail end indicates the initial positions at 15,820 . Since the aspect ratio decreased from 1.74 to 1.28 during this period, the picture displays the heart of the R-to-S transition. Briefly, the upper half of the crystal shears from right to left, relative to the lower half. However, a shear of $15 \mathrm{deg}$ cannot accommodate the transition, which involves both a volume expansion of 7 pct and shuffling of the B atoms (tadpoles with solid circles). Consequently, there arises a substantial difference in atomic displacements between the two species. The inclined dash line in Figure 7 indicates the alignment that both A and B atoms would make in the $\mathrm{S}$ phase. Figure 8 portrays the reverse transition, $\mathrm{S}$ to $\mathrm{R}$, during the period from 15,950 to 15,960 . The aspect ratio increased from 1.22 to 1.50 during this time. The transition appears to be a typical tetragonal deformation of mixed signs, combined with shuffling displacements for the B atoms in the right half of the crystal.

\section{B. Three-Dimensional Case}

The transition between the rhombus and square phase in the previous two-dimensional simulation mimics an allotropic transition between hcp and fcc cobalt observed during mechanical alloying (Figure 4). In order to see more realistic structural changes, including crystalline-to-glass transitions, the work is extended to a three-dimensional case. For atomic interactions, both the LJ potential and a semi-empirical, tight-binding (TB) potential are used. The TB potential is adopted from the work of Hsieh et al. ${ }^{[29]}$ and is given by

$$
\begin{aligned}
\Phi_{i}=- & {\left[\sum_{j=1}^{N} \xi_{i j}^{2} \exp \left\{-2 q_{i j}\left(\frac{r_{i j}}{d_{i j}}-1\right)\right\}\right]^{1 / 2} } \\
& +\sum_{j=1}^{N} A_{i j} \exp \left\{-p_{i j}\left(\frac{r_{i j}}{d_{i j}}-1\right)\right\}
\end{aligned}
$$

where $\xi_{i j}$ is an effective hopping parameter characterizing the second moment of the $d$-band state, $d_{i j}$ is the first-nearest-neighbor distance, and $A_{i j}$ is a BornMayer-type repulsive energy coefficient. The values of the parameters are listed in Table IV, in which all units 
Table III. Potential Parameters for Atomic Interactions for Two-Dimensional Study

\begin{tabular}{llcc}
\hline Parameter & A-A & A-B & B-B \\
\hline$\varepsilon_{i j}$ & 1.140 & 0.210 & 1.000 \\
$\sigma_{i j}$ & 0.900 & 0.600 & 0.865 \\
Mass & 3.5 & - & 1.0 \\
\hline
\end{tabular}

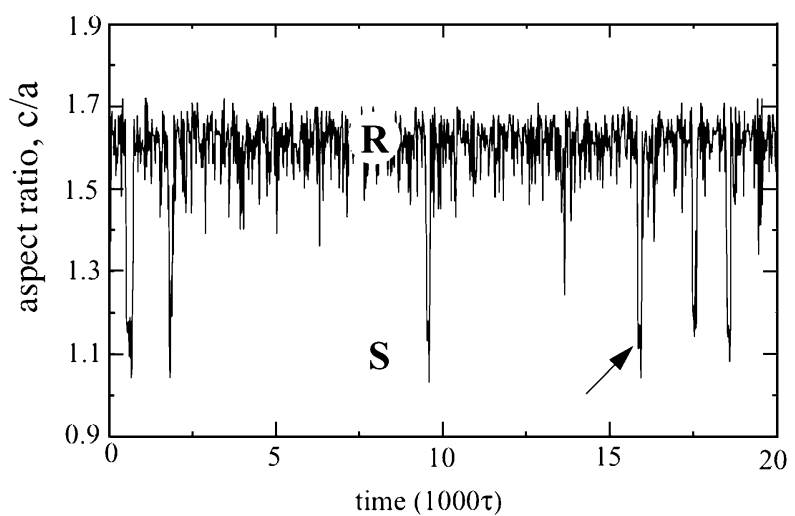

(a)

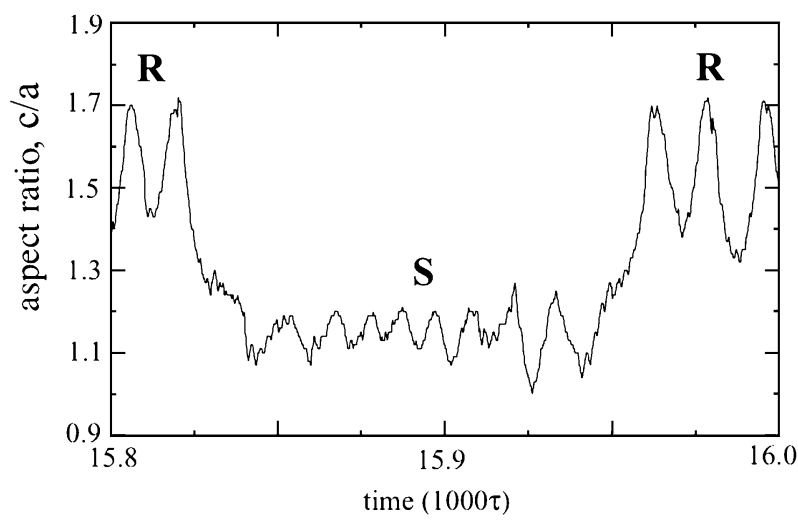

(b)

Fig. 6 - Structural changes of the 57-atom nanocrystal during milling. (a) shows the aspect ratio, $c / a$, of the crystal during the milling period from 0 to 20,000, and $(b)$ details the changes during the time between 15,800 and 16,000, arrow-marked in (a).

are reduced with length $\sigma=10^{-10} \mathrm{~m}$, mass $M=$ $5.8 \times 10^{-26} \mathrm{~kg}$, and energy $\varepsilon=2.5 \times 10^{-19} \mathrm{~J}$. The LJ parameters, $\varepsilon_{i j}$ and $\sigma_{i j}$ of Eq. [13], are simply equated to $\xi_{i j}$ and $d_{i j}$ of the TB case. Because of a strong attractive interaction between $\mathrm{Ni}$ and $\mathrm{Zr}$, i.e., $\xi_{\mathrm{Ni}-\mathrm{Zr}}>\left(\xi_{\mathrm{Ni}-\mathrm{Ni}}+\right.$ $\left.\xi_{Z r-Z r}\right) / 2$, it is an ideal binary system for studying the deformation behavior of an ordered CsCl-type B2 structure. However, the real crystal structure of $\mathrm{Ni}_{50^{-}}$ $\mathrm{Zr}_{50}$ is $\mathrm{B} 33$, although a number of intermetallic compounds of equicomposition, such as $\mathrm{Co}_{50}-\mathrm{Zr}_{50}$, belong to B2. Therefore, the two potentials represent, at best, a model B2 crystal. The cutoff distance is set at $0.6 \mathrm{~nm}$, which allows a given atom to interact with up to $\sim 60$ neighbors. Simulation temperatures are maintained at $300 \pm 5 \mathrm{~K}$ through a scaled velocity Verlet algorithm.

Metal powders pounded between two grinding balls undergo both compressing and shearing deformation,

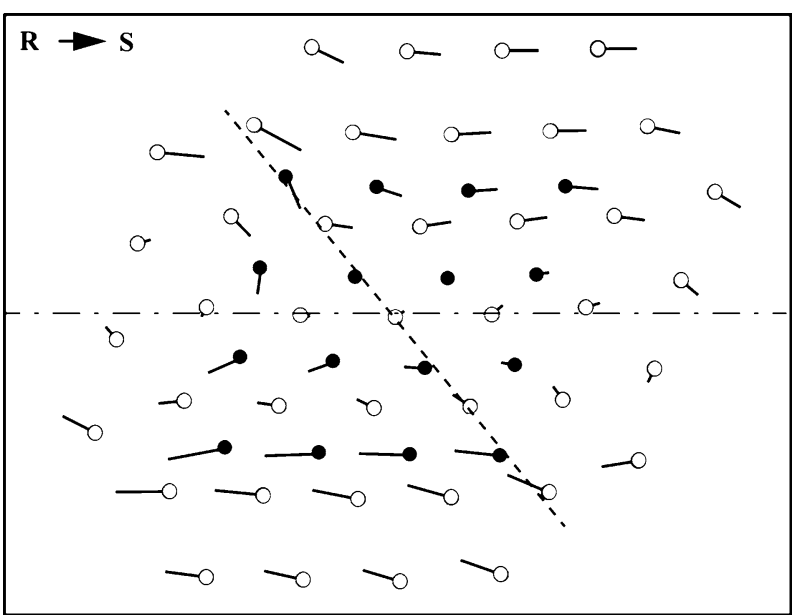

Fig. 7-An R-to-S transition process showing cooperative atomic displacements from the milling time, 15,820, to the time, 15,830. The head of each tadpole indicates the atomic position at 15,830 , and its tail end marks the initial position.

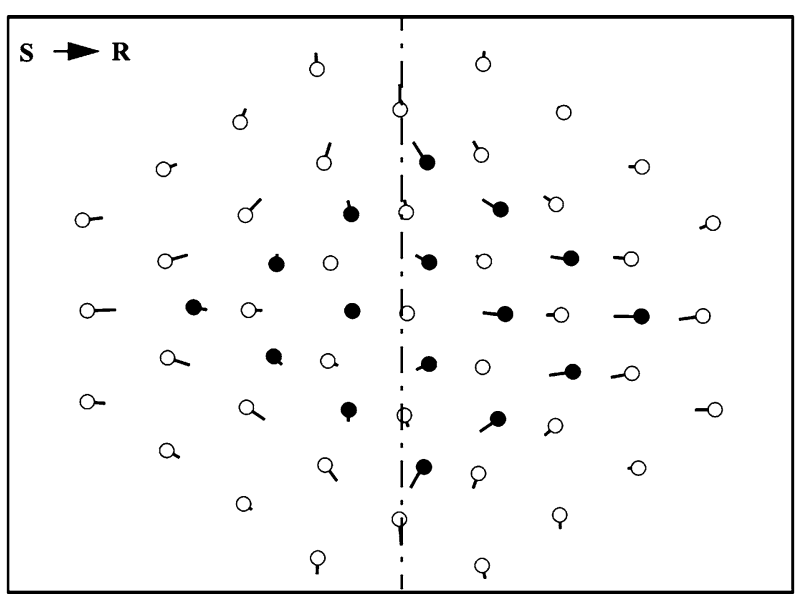

Fig. 8-A S-to-R transition process showing cooperative atomic displacements from the milling time, 15,950, to the time, 15,960. The head of each tadpole indicates the atomic position at 15,960, and its tail end marks the initial position.

which are sketched in Figure 9. In the compressing deformation mode, a periodic boundary condition is imposed along the loading axis, while a free surface is maintained for the other perpendicular directions. The periodic boundary condition along the $z$-axis allows atomic momentum transfer without undue influence of the loading machine surface. Alternatively, periodic boundary conditions can also be imposed, if a bulklike sample is desired, along the $x$ and $y$ directions. However, the presence of a free surface permits, even in a small sample, either twinning or bending, which occurs in real macroscopic metallic powders. Because the loading is simple, compressive deformation mode is widely used in the literature. ${ }^{[29,30]}$

In shearing deformation, however, the influence of the loading machine surface is not as simple as in compressive deformation. Fixed boundary conditions are often 
Table IV. Potential Parameters for Atomic Interactions for Three-Dimensional Study

\begin{tabular}{lccc}
\hline Parameter & $\mathrm{Ni}-\mathrm{Ni}$ & $\mathrm{Ni}-\mathrm{Zr}$ & $\mathrm{Zr}-\mathrm{Zr}$ \\
\hline$\xi_{i j}$ & 0.6857 & 1.3707 & 1.4604 \\
$q_{i j}$ & 1.1890 & 2.2300 & 2.2490 \\
$d_{i j}$ & 2.4900 & 2.7610 & 3.1790 \\
$A_{i j}$ & 0.0241 & 0.1388 & 0.1239 \\
$p_{i j}$ & 16.999 & 8.3600 & 9.3000 \\
$\varepsilon_{i j}$ & 0.6857 & 1.3708 & 1.4604 \\
$\sigma_{i j}$ & 2.4900 & 2.7610 & 3.1790 \\
mass & 1.6801 & - & 2.6103 \\
\hline
\end{tabular}

used at both the bottom and the top surfaces along the $z$ direction, but they can cause undue influence on the momentum transfer for the atoms located in the proximity of the load surfaces, unless the sample dimension is very large ${ }^{[31,32]}$ As sketched in Figure 9(b), the sandwich-type periodic boundary condition with a pivoted center line permits sheared atomic motions with correct momentum transfer, and thus appears to alleviate the modeling difficulty, as will be demonstrated in the results. It is important that a microsample in a simulation should mirror a macroscopic system as correctly as possible.

Figure 10 shows the radial distribution function (RDF), $g(r)$, for a $\mathrm{B} 2 \mathrm{Ni}-\mathrm{Zr}$ crystal undergoing compressive deformation at a constant strain rate, $\dot{\varepsilon}=0.01 / \mathrm{ps}$ and at $T=300 \mathrm{~K}$. The computation cell consists of $1130 \mathrm{Ni}$ and $1120 \mathrm{Zr}$ atoms and has an initial periodic length equal to $\sim 3.21 \mathrm{~nm}$ along the $z$-direction. Due to the free surface, the atom numbers are not even and they are allowed to interact through the TB potential. The $g(r)$ indicates the probability of finding atoms at a distance $r$ and is a measure for a dynamic structural factor. ${ }^{[30]}$ Thus, at zero deformation with $\varepsilon=0$, the RDF should display peaks at the first, second, third, ... neighbor positions consistent with the

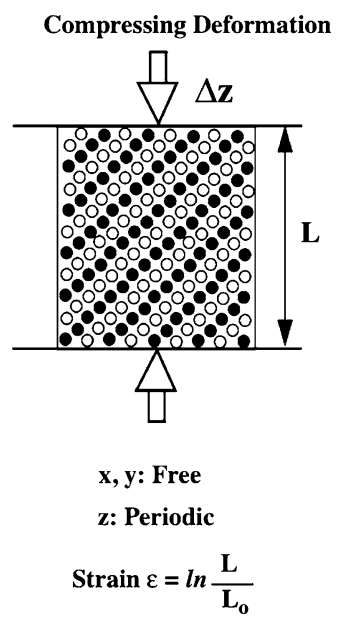

(a)

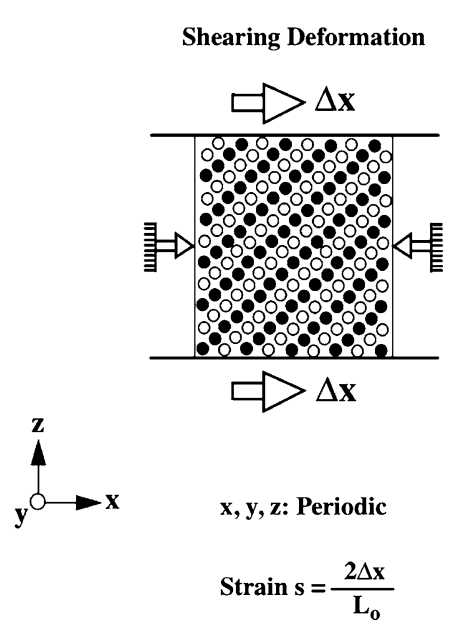

(b)
Fig. 9-Deformation modes: (a) compressive and $(b)$ shearing deformation. "Periodic" indicates periodic boundary conditions and "Free" is for a free surface.

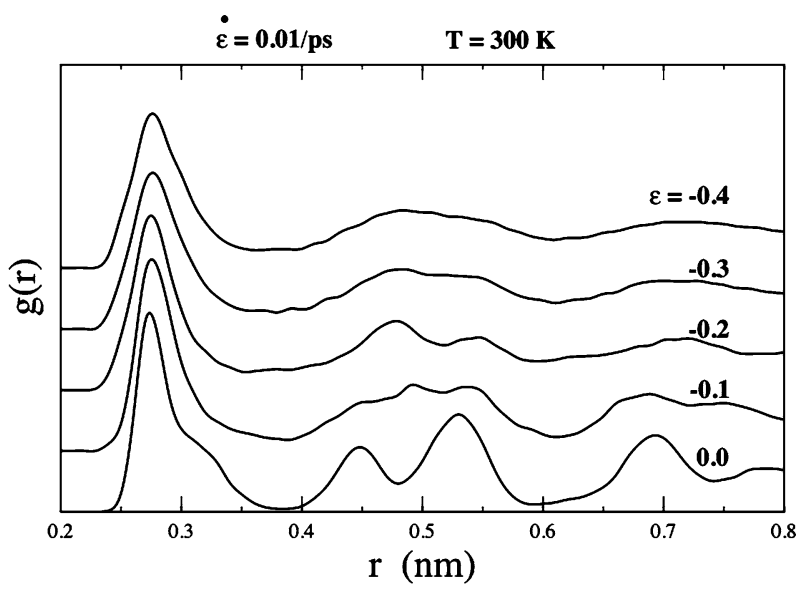

Fig. $10-\mathrm{RDF}, g(r)$, at varying strains, $\varepsilon$, for a crystal with $\mathrm{Ni}=$ 1130 and $\mathrm{Zr}=1120$ atoms interacting through the TB potential. The initial structure is a $\mathrm{B} 2$ crystal undergoing compressive deformation at a constant strain rate, $\dot{\varepsilon}=0.01 / \mathrm{ps}$, and at $T=300 \mathrm{~K}$.

B2 structure. The peaks on the $\varepsilon=0$ curve are somewhat broadened, due to the effects of both free surface and thermal vibrations. Although the peaks are not sharp, the initial crystalline nature of the sample is clearly shown in the first picture of Figure 11, where atomic positions are projected onto an $x-y$ plane perpendicular to the compressive axis. The $\mathrm{Ni}$ and $\mathrm{Zr}$ are marked with $\mathrm{x}$ and $\mathrm{o}$.

As the amount of compressive strain, $\varepsilon$, increases, all other peaks, except for the first peak, disappear or merge with each other, indicating that the crystalline

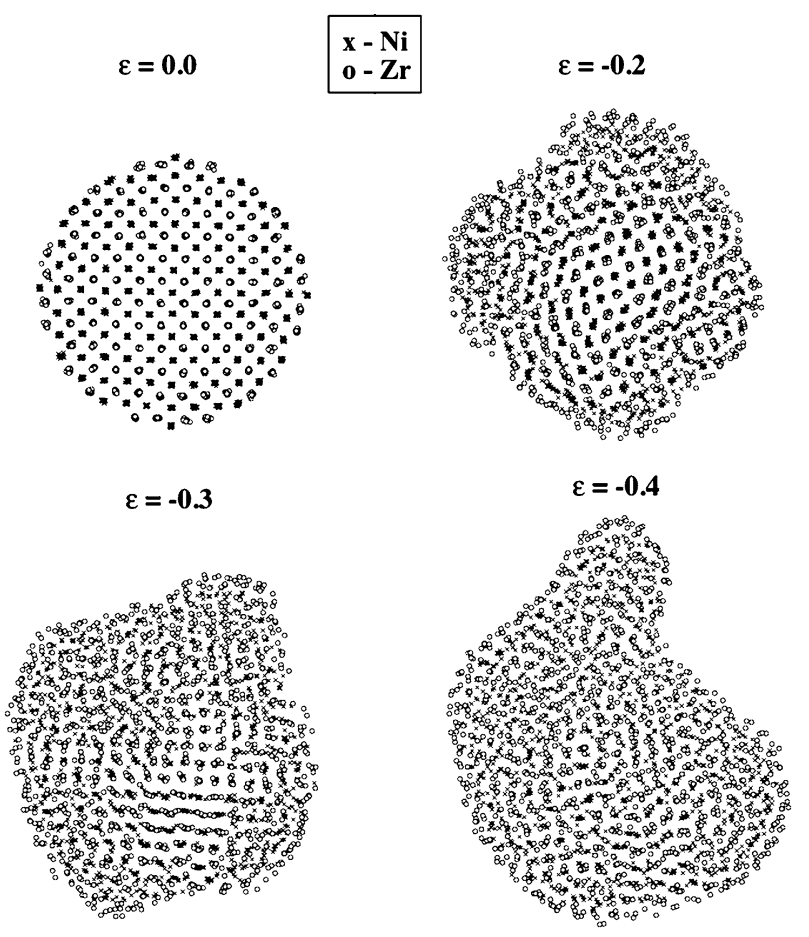

Fig. $11-$ Views of atomic positions projected onto $x-y$ planes perpendicular to the compression axis for the $\mathrm{TB}$ crystal with $\mathrm{Ni}=$ 1130 and $\mathrm{Zr}=1120$, undergoing compressive deformation at a constant strain rate, $\dot{\varepsilon}=0.01 / \mathrm{ps}$, and at $T=300 \mathrm{~K}$. 


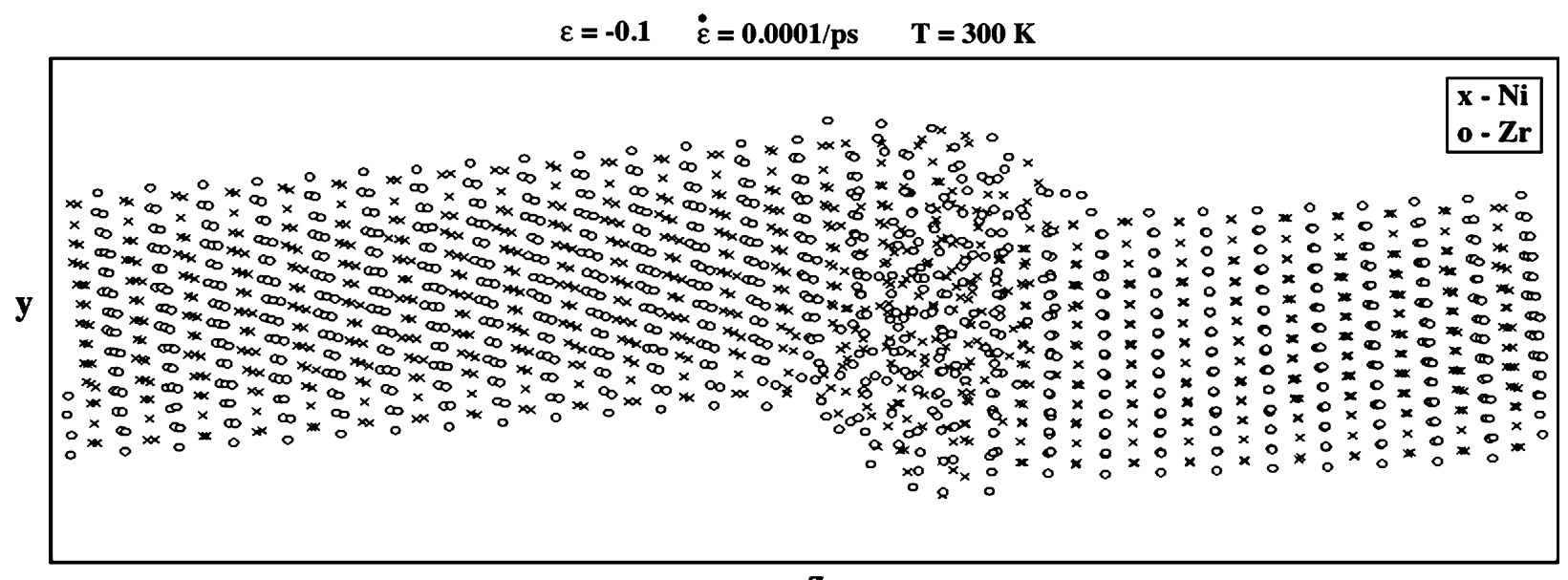

$\mathbf{z}$

Fig. 12-View of atomic positions projected onto $y$ - $z$ plane perpendicular to the compression axis for an $\mathrm{LJ}$ crystal with $\mathrm{Ni}=870$ and $\mathrm{Zr}=$ 960, undergoing a kinking at a strain $\varepsilon=-0.1$, at $\dot{\varepsilon}=0.0001 / \mathrm{ps}$ and $T=300 \mathrm{~K}$. The faulting region becomes a nucleation site for a glass phase.

nature is being lost. Variations in the $g(r)$ curves of Figure 10 are consistent with the pictures of the atomic positions in Figure 11. For this particular sample, the loss of crystallinity is shown to begin at the free surface, and the glass phase grows into the interior of the crystal. Eventually, at a strain $\varepsilon=-0.4$, there is no crystalline phase. Furthermore, the initial circular section is gone and the punching-bag-like cross section at $\varepsilon=-0.4$ suggests the work of dislocations or disclinations during the compressive deformation, although the details of these defects are not examined. When a sample is relatively slender, a kinking or a twinning is observed, as shown in Figure 12, in which the faulting region becomes a nucleation site for a glass phase.

Structural changes under shearing deformation show a strain rate dependence more sensitive than is shown under compressive deformation. Figure 13 shows RDF

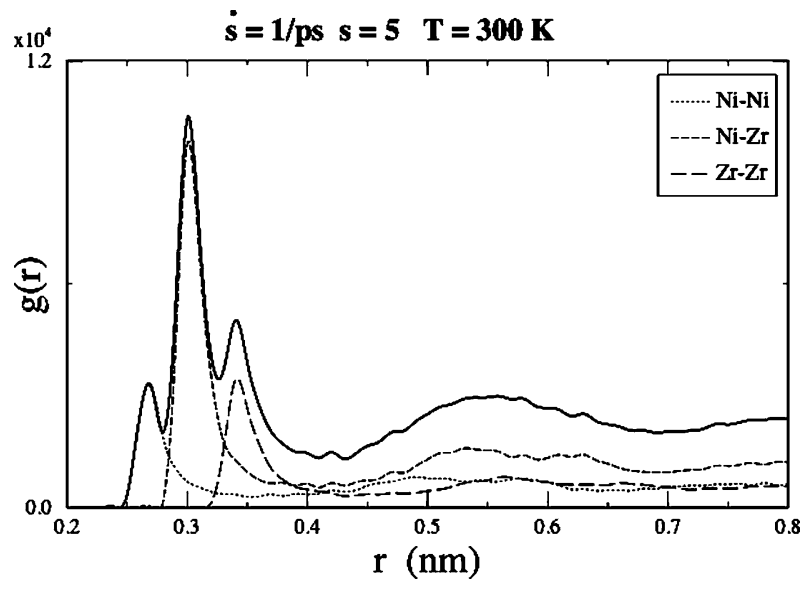

Fig. 13-RDF, $g(r)$, of an LJ crystal sheared to $s=5$ at a shear strain rate, $\dot{s}=1 / \mathrm{ps}$, and at $T=300 \mathrm{~K}$. The computation cell has $\mathrm{Ni}=2000$ and $\mathrm{Zr}=2000$ atoms interacting through the LJ potential. The top solid curve indicates the distribution function accounting for all the atoms. No other distinctive peaks, except for the three subdivided peaks for the first neighbors, indicate a glass phase. curves for a LJ B2 crystal of $\mathrm{Ni}=2000$ and $\mathrm{Zr}=2000$ at a shear strain, $s=5$, deformed at $\dot{s}=1 / \mathrm{ps}$ and $T=300 \mathrm{~K}$. The top solid curve indicates the distribution function accounting for all the atoms. The three nonsolid curves - dotted, dashed, and long-dashed - are for $\mathrm{Ni}-\mathrm{Ni}, \mathrm{Ni}-\mathrm{Zr}$, and $\mathrm{Zr}-\mathrm{Zr}$ distribution functions, respectively. The $x-z$ projected views of atomic positions, i.e., the views along the shear direction, are displayed in Figure 14 for two strains, $s=1$ on the top and $s=5$ on the bottom. The atomic projection at $s=5$ indicates a glass phase in agreement with the RDF curves of Figure 13. Compared to the TB case, the LJ potential has stronger attraction between the $\mathrm{Ni}$ and $\mathrm{Zr}$ atoms. This, in turn, causes the first-nearest-neighbor peak to split into three subpeaks- $-\mathrm{Ni}-\mathrm{Ni}, \mathrm{Ni}-\mathrm{Zr}$, and $\mathrm{Zr}$ $\mathrm{Zr}$ - even in a glass phase. Clearly, the two potentials are quite different, the LJ case having a stronger atomic interaction and greater atomic size difference than the TB case, which shows no split in the peak for the nearest neighbors in Figure 10. The atomic positions at $s=1$ in Figure 14 demonstrate that the system is divided roughly into two phases - a crystalline phase in the regions where atomic shear velocity is relatively low, i.e., the central and the left and right edge regions along $z$, and a glass phase in between the crystalline phases. As the shear strain increases, however, all the crystalline phase converts to the glass phase, as manifested in the bottom picture of Figure 14 with $s=5$.

What kind of structural changes are expected at very high shear strain rates? It seems that some answers can be provided through Figures 15 and 16. Figure 15 shows the RDF curves for the LJ B2 crystal of $\mathrm{Ni}=2000$ and $\mathrm{Zr}=2000$ at a shear strain, $s=80$, deformed at $\dot{s}=10 / \mathrm{ps}$, and $T=300 \mathrm{~K}$. As before, the top solid curve indicates the distribution accounting for all the atoms, and the three nonsolid curves are for the $\mathrm{Ni}-\mathrm{Ni}$, $\mathrm{Ni}-\mathrm{Zr}$, and $\mathrm{Zr}-\mathrm{Zr}$ distributions. A noticeable feature at this high strain of $s=80$ is that there are distinctive peaks at large distances, $r=0.52,0.58 \mathrm{~nm}, \ldots$. This, of course, suggests that the new structure is no longer 


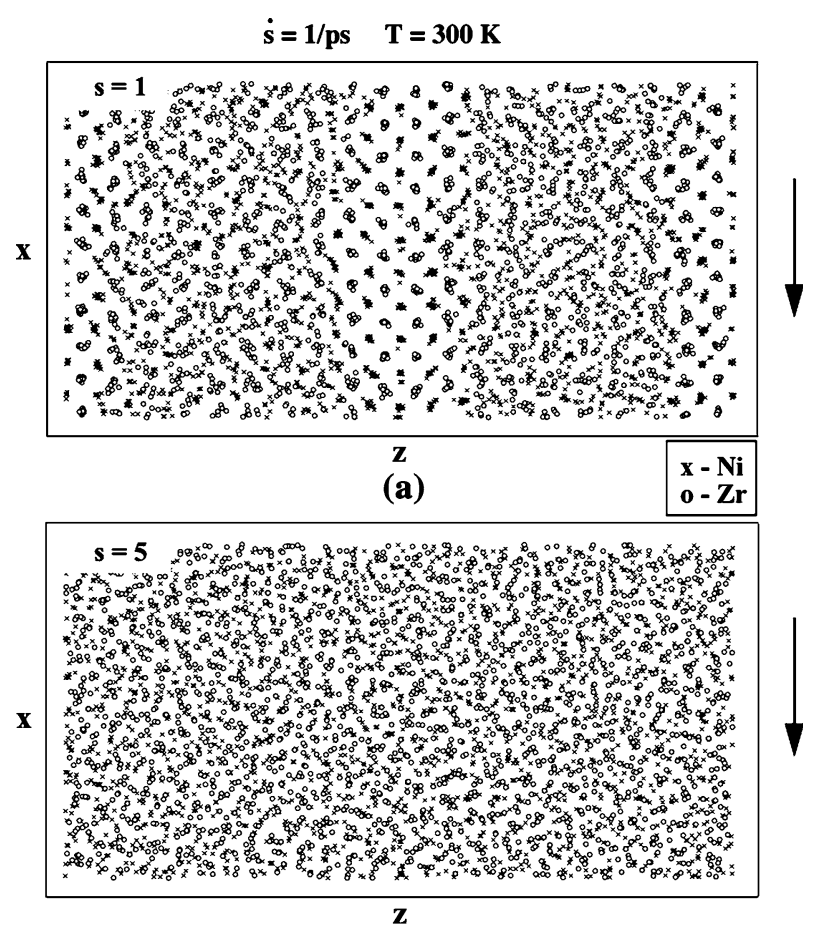

(b)

Fig. 14-Views of atomic positions projected onto $x-z$ planes parallel to the shear direction for the $\mathrm{LJ}$ crystal with $\mathrm{Ni}=2000$ and $\mathrm{Zr}=$ 2000 undergoing shear deformation at $\dot{s}=1 / \mathrm{ps}$, and at $T=300 \mathrm{~K}$. The downward arrowheads indicate shear direction. (a) Shear strain, $s=1$, and $(b) s=5$.

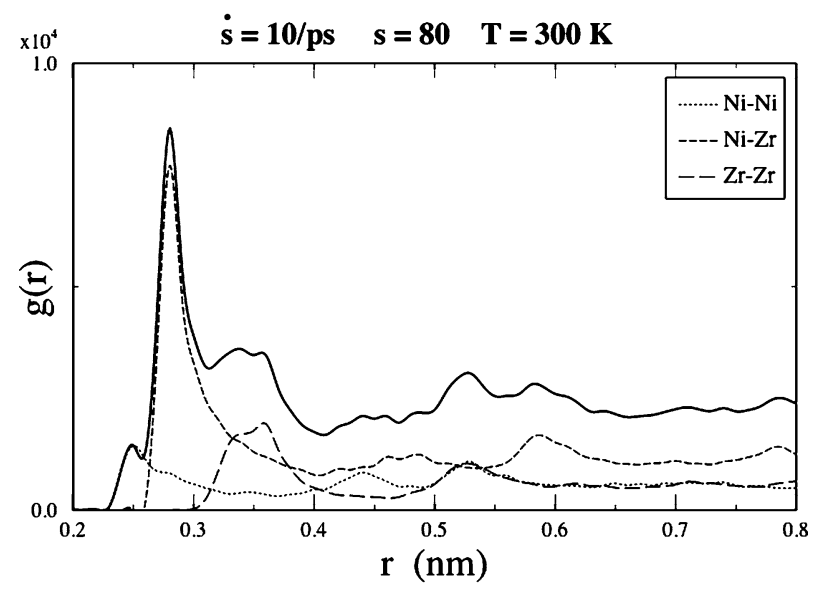

Fig. 15-RDF, $g(r)$, of an LJ crystal sheared to $s=80$ at a shear strain rate, $\dot{s}=10 / \mathrm{ps}$, and at $T=300 \mathrm{~K}$. The top solid curve indicates the distribution function accounting for all the atoms. The distinctive peaks at large $r=0.52$ and $0.58 \mathrm{~nm}$ indicate that the structure is not a glass phase.

amorphous. Indeed, atomic positions reveal that the phase has an ordered structure. Figure 16 shows, at the top, an $x-z$ plane view parallel to the shear direction and, at the bottom, a $y-z$ plane view perpendicular to the shear direction. At first glance, the $x-z$ view seems to bear no long-range order, but a careful examination displays a layered structure made of alternating rows of $\mathrm{Ni}$ and $\mathrm{Zr}$ atoms parallel to the shear direction. The $y$-z view
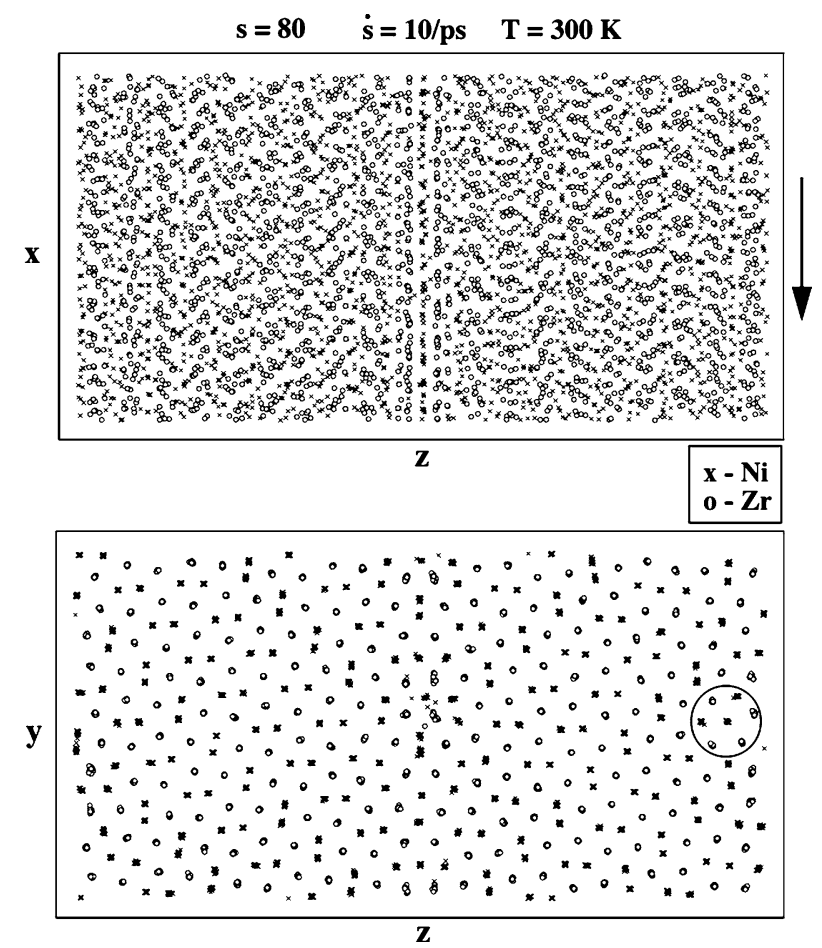

Fig. 16-Views of atomic positions projected onto $x-z$ and $y$-z planes for an LJ crystal sheared to $s=80$, at $\dot{s}=10 / \mathrm{ps}$ and at $T=300 \mathrm{~K}$. Top; an $x-z$ plane view parallel to the shear direction, marked with an arrow head. Bottom: a $y-z$ plane view perpendicular to the shear direction, and the circle on the right indicates a unit cell. Note a string phase created at this high strain rate deformation.

perpendicular to the shear direction discloses not a random but a two-dimensional ordered structure with 5 to 7 nearest neighbors at equidistance: a circle is drawn to indicate a unit cell. Summing up the two, the $x-z$ and $y-z$ atomic views, it seems clear that a high shear strain rate can create a string phase, even on an atomic scale. In the field of colloids, in which the particle scales are of $\sim 100 \mathrm{~nm}$, the dynamical changes taking place during extreme shear thinning are well characterized by the linear lines of molecules forming along the shear direction - the strings of molecules pack into a hexagonal lattice, when viewed perpendicular to the shear direction - and this is termed a "string phase." ${ }^{, 33]}$ The current work demonstrates the possibility of forming a string phase on an atomic scale at a high shear strain rate.

\section{DISCUSSION}

The results of the molecular dynamics study presented here are only preliminary, and further work is needed in order to mimic realistic cyclical phase transformations occurring in a driven system. For example, with a large crystallite, one could expect crystalline defects, such as a two-dimensional square-rhombus interface or dislocations that may play crucial roles during cyclical transformations. The square-rhombus phase transition is an ideal case representing no kinetic constraint in the phenomenological description of Courtney and Lee. ${ }^{[3]}$ 
For the three-dimensional compressive deformation mode, there have been numerous simulation results displaying transitions between the crystalline and glass phases, and the current work confirms that a glass phase can be nucleated from a free surface or a faulted region of a twining or a kinking.

For shear deformation, a new simulation scheme is introduced, to allow a correct atomic momentum transfer without undue influence from the loading machine surface. In many of the earlier investigations, fixed boundary conditions are used for the interface between the computation cell and the loading machine: it is simple, but the atomic momentum calculation in the neighborhood of the loading machine surface is apt to transfer incorrectly. ${ }^{[32]}$ This can be viewed with Figure 9(b): imagine that the top and the bottom horizontal lines represent the interface planes between the loading machine and the computation cell. The atomic momenta along the $z$ direction should diminish to zero, as atoms approach the machine wall. As a consequence, these "frozen" atoms at the interface region influence the dynamics of the interior atoms, unless the cell size along the $z$ direction is very large. By no means is the new scheme perfect. As portrayed in Figure 14(a), it creates an extra plane with zero shear velocity at the center and, thus, a large cell size along the $z$ direction is preferred. Nevertheless, it mimics momentum transfer compatible to the applied shear strain rate, by alleviating the unwarranted influence of the loading machine surface.

In the field of colloids with monodisperse particles $10 \mathrm{~nm}$ to $1 \mu \mathrm{m}$ in diameter, there has been great progress on phase transition behavior in particle arrangement. ${ }^{[33,34]}$ For example, using synchrotron X-ray scattering techniques, Sirota et al. ${ }^{[34]}$ found that as the volume fraction of polystyrene spheres in the liquid mixture of methanol and water increases, the system undergoes structural changes from liquidlike to a colloidal crystal of, first, bcc and, then, fcc. Furthermore, the colloidal viscosity is also studied and is found to be strongly dependent on the applied shear strain rate, exhibiting Newtonian as well as non-Newtonian behavior. At high strain rates, shear thinning, i.e., a drastic reduction in viscosity, is observed, which indicates dynamic structures with stream lines of colloidal particles along the shear direction. It is interesting to note that a high shear strain rate can create a solid-state string phase even on an atomic scale, as demonstrated in Figure 16.

It should be noted that improved simulations are necessary, in that a constant atomic volume is used for the shear deformation. In reality, deformation involves both compression as well as shearing, and thus, the molar volume is not conserved. A better scheme must reflect both modes of deformation, perhaps even a hydrostatic pressure to mimic a ball-milling process. In our simulation, no nucleation of the three-dimensional crystalline phase out of the glass phase is observed, although one might consider that the string phase shown in Figure 16 could be a precursor of a three-dimensional crystalline phase. A glass phase represents a system of nonergodicity, which means that a time average does not necessarily represent an ensemble average.
Therefore, it is not a surprise that simulations with only a few thousand atoms fail to show crystallization in a time of less than a nanosecond. In other words, sampling of a very minute sequence of a tiny amorphous cluster has too low a probability to exhibit such a transition.

The DDE analyses on the flow curve, for metals with low stacking fault energies, demonstrate that a cyclical or oscillatory behavior of certain materials properties - stress or free energy - in a driven system can be described with a delay or drag nature, due to diffusion. In Table II, the incubation time for the nucleation for recrystallization is calculated with a linear relationship, $\tau\left(=\varepsilon_{n} / \dot{\varepsilon}\right)$, and the results display a significant increase from 0.09 to 45.5 seconds, as shown in the rightmost column. The incubation time, $\tau$, is equal to $\sim l^{2} / D$, where $l$ is the mean diffusion distance and $D$ is the selfdiffusion coefficient. ${ }^{[35]}$ If we take $l$ as $d / 2, d$ being the mean subgrain size, and D $\sim 7.7 \times 10^{-16} \mathrm{~m}^{2} / \mathrm{s}$ (for $\gamma-\mathrm{Fe}$ at $\left.1100{ }^{\circ} \mathrm{C}^{[36]}\right)$, the incubation times from 0.09 to 45.5 seconds suggest mean subgrain sizes from about 17 to $374 \mathrm{~nm}$. The increase in the subgrain size with a decrease in the strain rate, $\dot{\varepsilon}$, simply manifests the decrease in the steady-state stress, $\omega$. For the alloys with high stacking fault energies, as noted earlier, easy cross slip or climb is sufficient to reduce dislocation densities below the level for the onset of dynamic recrystallization, and thus there arise no multiple peaks in the flow curve. For such a case, the incubation time, $\tau$, becomes on the order of $\sim 0.1$ seconds, if $l$ is taken as the mean dislocation spacing, with $\rho \sim 10^{16} / \mathrm{m}^{2}$. Therefore, $\varepsilon_{n}$ would be $\sim 10^{-4}$ at a typical slow strain rate $\dot{\varepsilon}=10^{-3} / \mathrm{s}$, which is too small to cause oscillations on the flow curve for those alloys or metals. A similar conjecture is applied to the glass phase, which competes against the crystalline phase during a ball-milling process (Figure 3 ).

Cyclical phase transformations can be understood in terms of reaction-like competition between participant phases. For example, in a recent work of Johnson et al., ${ }^{[37]}$ thermodynamic balance equations are expressed to describe the time evolution of two competing phases undergoing ball milling. The state of the microstructure is then determined by the phase fraction, the deformation state of each phase, and the homogeneous temperature. One of the steady-state solutions shows spiral points suggesting cyclic amorphization behavior, in agreement with the DDE results of Eq. [12]. Beyond phenomenological treatments such as DDE, however, it is desirable to see how atomistic mechanics is working in cyclical transitions. Accordingly, some molecular dynamics simulations are initiated in this work, but much work is clearly needed to answer many of the puzzling questions associated with cyclical phase transformations in driven systems.

\section{SUMMARY}

Cyclical phase transformations occurring in driven systems, such as hot working or ball milling, are described in terms of a minimization process of the free energies of participant phases. Based on the oscillatory flow behavior of metals with low stacking fault energies 
during hot working, it is argued that a ductile crystalline phase sustains undulation in its free energy, due to the alternate succession of work-hardening and work-softening mechanisms. A time-dependent free energy function is then obtained by solving a DDE, which accounts for a time lag due to diffusion. The oscillatory behavior of the function is shown to depend both on the recovery capacity and the delay time of a given phase, and thus allows us to understand the systems that would experience cyclical phase transformations. In an attempt to understand cyclical transitions on an atomistic scale, molecular dynamics simulations are also studied, for both two-dimensional and three-dimensional crystals undergoing shear deformation. The two-dimensional results of a nanocrystal shows cyclical transitions between an equilibrium rhombus and a nonequilibrium square phase. The results of the three-dimensional simulations show crystalline-to-glass transitions at high strain rates, and the nature of the transition is either homogeneous or heterogeneous, depending on the availability of defects, such as free surface or stacking faults. However, very high shear strain rates produce a string phase made of linear lines of atoms, when viewed along the shear direction, but of a latticelike network, when viewed perpendicular to the shear direction.

\section{ACKNOWLEDGMENTS}

The work is prepared in memory of two great materials scientists, Drs. Hubert I. Aaronson and Thomas H. Courtney: for three decades, the author's life was profoundly enriched by these two gentlemen. The author is also grateful to Professor William C. Johnson for the delightful discussions during the course of the work.

\section{REFERENCES}

1. M. Sherif El-Eskandarany, K. Aoki, K. Sumiyama, and K. Suzuki: Metall. Mater. Trans. A, 1999, vol. 30A, p. 1877.

2. J.Y. Huang, Y.K. Wu, and H.Q. Ye: Acta Mater., 1996, vol. 44, p. 1201.

3. T.H. Courtney and J.K. Lee: Philos. Mag., 2005, vol. 85, p. 153.

4. J.K. Lee and W.C. Johnson: in Solid to Solid Phase Transformations in Inorganic Materials 2005, Vol. 1: Diffusional Transformations, J.M. Howe, D.E. Laughlin, J.K. Lee, U. Dahmen, and W.A. Soffa, eds., TMS, Warrendale, PA, 2005, p. 843.

5. M. Sherif El-Eskandarany, K. Aoki, K. Sumiyama, and K. Suzuki: Scripta Mater., 1997, vol. 36, p. 1001.
6. M. Sherif El-Eskandarany, K. Aoki, K. Sumiyama, and K. Suzuki: Appl. Phys. Lett., 1997, vol. 70, p. 1679.

7. M. Sherif El-Eskandarany and A. Inoue: Metall. Mater. Trans. A, 2002, vol. 33A, p. 2145.

8. T. Nagase and Y. Umakoshi: Mater. Trans., JIM, 2004, vol. 45, p. 13.

9. X. Zhang, H. Wang, R.O. Scattergood, J. Narayan, and C.C. Koch: Acta Mater., 2002, vol. 50, p. 3995.

10. Y.H. Zhao, K. Lu, and K. Zhang: Phys. Rev. B, 2002, vol. B66, p. 085404.

11. C.M. Sellars and W.J.McG. Tegart: Acta Mater., 1966, vol. 14, p. 1136.

12. C. Rossard: Metaux, 1960, vol. 35, p. 102, pp. 140 and 190.

13. M.J. Luton and C.M. Sellars: Acta Metall., 1969, vol. 17, p. 1033.

14. R. Sandstrom and R. Lagneborg: Acta Metall., 1975, vol. 23, p. 387.

15. A.D. Rollet, M.J. Luton, and D.J. Srolovitz: Acta Metall. Mater., 1992, vol. 40, p. 43.

16. P. Peczak and M.J. Luton: Philos. Mag. B., 1994, vol. 70, p. 817.

17. R.L. Goetz and V. Seetharaman: Scripta Mater., 1998, vol. 38, p. 405.

18. R. Ding and Z.X. Guo: Acta Mater., 2001, vol. 49, p. 3163.

19. V.G. Garcia, J.M. Cabrera, and J.M. Prado: Mater. Sci. Forum, 2004, vol. 467-470, p. 1181

20. V.G. Garcia: Ph.D. Thesis, Universitat Politecnica de Catalunya, 2004, http://www.tdx.cesca.es/TDX-0104105-09244/.

21. U.F. Kocks: Trans. ASME, J. Eng. Mater. Technol., 1976, vol. 98, p. 76.

22. I.R. Epstein: J. Chem. Phys., 1990, vol. 92, p. 1702.

23. H.J. McQueen and J.J. Jonas: in Treatise in Materials Science and Technology, Vol. 6: Plastic Deformation of Materials, R.J. Arsenault, ed., Academic Press, New York, NY, 1975, p. 392.

24. J.K. Lee: Mater. Sci. Forum, 2007, vol. 558-559, p. 441.

25. J.G. Lee, H. Mori, and H. Yasuda: Phys. Rev. B, 2002, vol. B66, p. 012105 .

26. O. Kastner: Continuum Mech. Thermodyn., 2003, vol. 15, p. 487.

27. O. Kastner: Continuum Mech. Thermodyn., 2006, vol. 18, p. 63.

28. J.K. Lee, H.K. Chang, and W.C. Johnson: Proc. 3rd Int. Conf. on Computational Modeling and Simulation of Materials, P. Vincenzini and A. Lami, eds., Techna Group Srl, 2004, p. 51.

29. P.J. Hsieh, Y.C. Lo, J.C. Huang, and S.P. Ju: Intermetallics, 2006, vol. 14, p. 924.

30. M.P. Allen and D.J. Tildesley: Computer Simulation of Liquids, Clarendon, Oxford, United Kingdom, 1987, p. 71

31. F. Delogu: Mater. Sci. Eng. A, 2006, vol. 426, p. 355.

32. D.M. Heyes: Physica B, 1985, vol. 131B, p. 217.

33. H.M. Laun, R. Bung, S. Hess, W. Loose, O. Hess, K. Hahn, E. Hadicke, R. Hingmann, F. Schmidt, and P. Lindner: J. Rheol., 1992, vol. 36, p. 743.

34. E.B. Sirota, H.D. Ou-Yang, S.K. Sinha, and P.M. Chaikin: Phys. Rev. Lett., 1989, vol. 62, p. 1524.

35. H.I. Aaronson and J.K. Lee: in Lectures on the Theory of Phase Transformations, H.I. Aaronson, ed., TMS, Warrendale, PA, 1999, 2nd ed., p. 165.

36. P. Shewmon: Diffusion in Solids, 2nd ed., TMS, Warrendale, PA, 1989, p. 78.

37. W.C. Johnson, J.K. Lee, and G.J. Shiflet: Acta Mater., 2006, vol. 54 , p. 5123 . 